\title{
Causal asymmetry across cultures: assigning causal roles in symmetric physical settings
}

\author{
Andrea Bender* and Sieghard Beller \\ Department of Psychology, Albert-Ludwigs-University, Freiburg, Germany
}

Edited by:

Mutsumi Imai, Keio University, Japan

Reviewed by:

Barbara C. Malt, Lehigh University, USA

Joseph A. Vandello, University of South Florida, USA

${ }^{*}$ Correspondence:

Andrea Bender, Department of

Psychology, Abteilung Allgemeine Psychologie,

Albert-Ludwigs-University,

Engelbergerstrasse 41, D-79085

Freiburg, Germany.

e-mail: bender@psychologie.uni-

freiburg.de
Causal cognition in the physical domain has been treated for a long time as if it were (1) objective and (2) independent of culture. Despite some evidence to the contrary, however, these implicit assumptions have been rarely ever explored systematically. While the pervasive tendency of people to consider one of two equally important entities as more important for bringing about an effect (as reported by White, 2006) meanwhile provides one type of counter-evidence for the first assumption, respective findings remained mute to the second. In order to scrutinize how robust such tendencies are across cultures and, if not, on which aspects of culture they may depend - we asked German and Tongan participants to assign prime causality in nine symmetric settings. For most settings, strong asymmetries in both cultures were found, but not always in the same direction, depending on the task content and by virtue of the multifaceted character of "culture." This indicates that causal asymmetries, while indeed being a robust phenomenon across cultures, are also modulated by task-specific properties (such as figure-ground relations), and are subject to cultural influences.

Keywords: causal cognition, culture, linguistic cues, agency, figure-ground distinction, physical domain, asymmetry bias

\section{INTRODUCTION}

Causality is one of the core concepts in our attempts to make sense of the world, and the explanations we come up with shape our judgments, emotions, and intentions. This renders causal cognition a key topic for the social as well as the cognitive sciences. However, despite an increasing awareness of the cultural constitution of cognition in various domains (e.g., Markus and Kitayama, 1991; Majid et al., 2004; Atran and Medin, 2008; Beller and Bender, 2008; Legare and Gelman, 2008; Bender et al., 2010a; Boroditsky and Gaby, 2010; Bender and Beller, 2011a; Fausey and Boroditsky, 2011), little is known about the impact of culture on causal cognition in the physical domain. While a plethora of insights into general principles and processes of causal cognition have been gathered (e.g., Talmy, 1988; Waldmann et al., 2006; Gopnik and Schulz, 2007; Wolff, 2007), respective research is still largely confined to European and US samples (cf. Arnett, 2008). After all, the physical world is the same for everybody and is open to direct inspection; indeed, a thorough understanding of it is essential for survival. These considerations may have motivated the assumption that perceiving physical causality should be largely objective and independent of culture (e.g., Michotte, 1963; Sperber et al., 1995; Spelke and Kinzler, 2007). However, unless we empirically scrutinize this assumption, we may not consider it to be valid (cf. Norenzayan and Heine, 2005; Atran and Medin, 2008; Henrich et al., 2010). While it might be true that people intuitively grasp causal relationships, at least in simple settings, it is an entirely different matter to assume that they understand and represent it in the same - and physically adequate - way. And in fact, a range of counter-arguments cast doubt on this latter assumption.

\section{HOW OBJECTIVE IS CAUSAL COGNITION?}

The very premise that physical causal cognition is based on objective observation, is contradicted not only by popular misconceptions, such as the impetus concept in motion (McCloskey, 1983; Hubbard and Favretto, 2003) or the valve model of how a thermostat functions (Kempton, 1986), but also by systematic biases. For instance, people tend to assign causal roles (i.e., cause vs. effect) even in strictly symmetric interactions. This effect has been described by Peter White (2006), who considers it to be the initial stage of the two-stage causal asymmetry bias (in the second stage, people tend to overestimate the impact of the cause object and underestimate that of the effect object). Such an asymmetric assignment of causal roles was experimentally demonstrated for a variety of collision events (White, 2007): When a moving object A hits a resting object $\mathrm{B}$ that then starts to move, the scene is generally interpreted as a launching event caused by object A. An alternative, yet still asymmetric reading could be that of a braking event, in which object B stops the movement of object A. However, for most people, this reading is neither obvious nor preferable.

The asymmetry bias is considered a general feature of causal cognition, affecting most of what people perceive, believe, and linguistically express with regard to causal relations (White, 2006). In fact, the bias appears to be so pervasive that it even restricts research questions and methods (White, 2006; Beller and Bender, in preparation), thus raising the issue of how robust it is across cultures.

\section{HOW INVARIABLE IS CAUSAL COGNITION?}

There are also reasonable challenges for the assumption that physical causal cognition is invariable across cultures. One piece of 
counter-evidence stems from research on causal attribution and on attribution tendencies such as the "correspondence bias." The correspondence bias leads people to attribute observed behavior to personal dispositions rather than situational factors. However, this bias is much more pronounced among US Americans who are reported as being more individualistic oriented than among Chinese people who are reported as being more collectivistic oriented (e.g., Morris and Peng, 1994; Norenzayan and Nisbett, 2000). Extending studies on such cross-cultural differences from the social to the physical domain, Peng and Knowles (2003) found respective differences between US-American and Chinese causal attributions for three out of eight physical scenarios.

Although it remains somewhat controversial whether the correspondence bias provides an unambiguous case of biasing (Malle, 1999) - and despite the fact that White (2006) dismisses it as a social instance of the causal asymmetry bias - these findings indicate at least that even in the physical domain, causal cognition may be affected by culture. However, "culture" is not a homogenous entity that could be located on a single dimension such as individualistic vs. collectivistic orientation, but is rather composed of innumerous aspects that may shift behavior into diverse directions (e.g., Atran and Medin, 2008; Beller et al., 2009a; Bender and Beller, 2011b). To be sure, the cultural aspects relevant for causal cognition do include social values and norms (which are partly reflected in self-concept and social orientation), but they do also encompass components such as culture-specific concepts and folk theories or linguistic habits.

It is meanwhile well established that people's causal assessments can be influenced by the information activated, for instance, by linguistic cueing, by adding (irrelevant) covariation information, or by substituting content elements in formally analogous tasks. More specifically, the choice of particular verbs may affect people's decisions due to the implicit causality carried by the verb (e.g., "A betrays B" invites more causal attribution for A, whereas "A praises B" invites more causal attribution for B; cf. Majid et al., 2007, and see Wolff et al., 2005). Similar effects can be obtained by assigning the thematic roles of agent and patient differently (Beller et al., 2009b). Also, providing covariation information for instance, by declaring one of two causes as given and the other as added - leads people to assume that the latter has more causal weight than the former (Cheng, 1997; Kuhnmünch and Beller, 2005; Beller and Bender, in preparation). Content, finally, has been documented for decades to affect performance in reasoning tasks, both by facilitating the valid conclusion when it converges with the formal structure of the task, and by suppressing it when the two diverge (Cummins, 1995; Beller and Kuhnmünch, 2007). These latter findings also suggest that people habitually access background knowledge, which then modulates the reasoning process (Beller and Spada, 2003; Klauer et al., 2010). As composition and availability of such knowledge is culture-specific, it should give rise to cultural differences in causal inferences (cf. Atran and Medin, 2008).

Crucially, there is no reason to assume that this multifaceted cultural background of participants should give rise to one coherent pattern of cultural differences in causal cognition. Rather, it is more likely that these different factors may have distinct effects, pointing into diverging directions.

\section{DESIGN AND GOALS OF THE CURRENT STUDY}

Despite this preliminary evidence to the contrary, hardly any of the previous studies empirically tested the implicit assumptions of objectivity and invariability. Addressing these issues was the intention of our cross-cultural screening study, which aimed at explicitly assessing whether causal asymmetries are affected by culture and, if so, which factors apart from a general attribution tendency notably linguistic cueing, covariation information, and content might modulate this effect.

In order to be able to detect an asymmetric role assignment, a task is required in which two entities do interact symmetrically to bring about an effect. Take, for instance, the floating task from Beller et al. (2009b):

Is the fact that a piece of wood floats on water basically due to ...
(a) the piece of wood; (b) the water?

From a physical point of view, the floating depends on the ratio of the densities of wood and water; the answer, therefore, must be that the two entities are equally causative. In fact, the symmetry of this static setting should be more easily discernible by laypersons than the symmetry of the dynamic collision events used by White (2007), which strike people as asymmetric simply on temporal grounds. An asymmetric role assignment (e.g., a preference for the floater) with such static settings would therefore be an even stronger finding than with dynamic events.

Previous cross-cultural studies typically focused on differences pertaining to an individualistic vs. collectivistic orientation (or features related to this distinction; cf. Peng et al., 2001). While a large body of data indeed suggests that, on a general level, causal assignments might be correlated with these orientations, the study of Beller et al. (2009b) questions the exclusivity of this account: With floating settings like the one presented above, the general tendency to mark the floater (i.e., the "individual") as being the prime cause for the floating in a rather individualistic culture, but the carrier (i.e., the supportive "medium") in a more collectivistic culture, could be shown to be strongly modulated both by linguistic cues and task content. These findings suggest that the general mechanism for causal assignments includes processes of activating, selecting, and weighing up of different kinds of linguistic information and individual knowledge. However, the previous study was constrained to one type of setting (a floating setting), one linguistic variation (transitive vs. intransitive phrasing), one type of content variation (wood vs. oil as floater), and one type of response format (forced-choice). This set-up does not allow for broad generalizations, and it did only provide first hints for, but no conclusive interpretation of, the differences found. In this study, we therefore intended to probe a wider range of factors beyond general attribution tendencies and to explore their distinct effects.

As in Beller et al. (2009b), we compared participants from Germany and Tonga, a Polynesian island kingdom in the Southwest Pacific. In this culture, Christianity is prevailing since the proselytization in the nineteenth century, and scientific findings and theories are conveyed during school education. Yet, some of the traditional concepts pertaining to a dynamistic worldview still coexist with the introduced Western concepts (Bender, 2001; Bender and Beller, 2003). In the social domain, on the other hand, the 
social structure and fundamental cultural values remained largely unaffected. They are based on relative rank, a pronounced group orientation, and an emphasis on mutual support which, in turn, generate strong social cohesion and a large degree of heteronomy (Marcus, 1978; Morton, 1996; Evans, 2001; Bender et al., 2007). This pervasive influence of one's social environment on one's decisions and behavior adds not only to the external causes of behavior, but most likely also fosters an awareness of these external causes, thereby supporting situational attributions.

In line with this characterization, the Tongan culture differs from the German one on a wide range of dimensions, three of which are of particular interest here. First, whereas individualistic values are emphasized together with a rather independent selfconcept in Germany, collectivistic values are emphasized together with a rather interdependent self-concept in Tonga (Bender et al., 2007; Beller et al., 2009a). This renders the two cultures apt candidates for a comparison in the classical individualism/collectivism paradigm as they reflect the usual differences in social orientation, while at the same time providing a valuable extension to the preponderant American/Asian contrast. In addition, due to partial differences in folk-physical convictions, distinct effects of content may be expected. And finally, the two languages differ with regard to how they categorize agents and patients in causative constructions. German as a nominative-accusative language treats subjects of transitive and intransitive sentences alike (with the nominative case), while setting them apart from the objects of transitive sentences (in the accusative). Tongan as an ergative-absolutive language, on the other hand, treats subjects of intransitive sentences and objects of transitive sentences alike (with the absolute case), while subjects of transitive sentences are set apart by the ergative case (Chung, 1978; Duranti, 1994; Bender and Beller, 2003; and see Figure 1).

According to the individualism/collectivism paradigm (Peng and Knowles, 2003), one would expect causal asymmetries to be more pronounced in Germany than in Tonga as the collectivistic cultural orientation in Tonga should lead to a more balanced consideration of both entities. However, this general tendency might be modulated by other factors relevant for assessing causality.

One candidate factor is the linguistic marking of agency (cf. Beller et al., 2009b). Agency can be linguistically assigned to either entity in question by different means, for example, by using particular causal verbs, grammatical roles, active vs. passive voice (in German), or ergative vs. non-ergative constructions (in Tongan). If agency is a relevant linguistic cue for making causal role assignments then the linguistically marked agent should be preferred as prime cause. In the floating setting above, this would be the wood.

Another candidate for modulating the perception of causality is the figure-ground distinction. Suppose that in the setting above, floating resulted from pouring water into a bowl that contained a piece of wood. As the water is added to the scene, it might be considered the figure, while the wood, being there from the very beginning, provides the background. If being a figure is relevant for making causal role assignments then the water should be preferred as prime cause.

A second possibility for marking one entity as the figure is by adding covariation information (Cheng, 1997; Kuhnmünch and

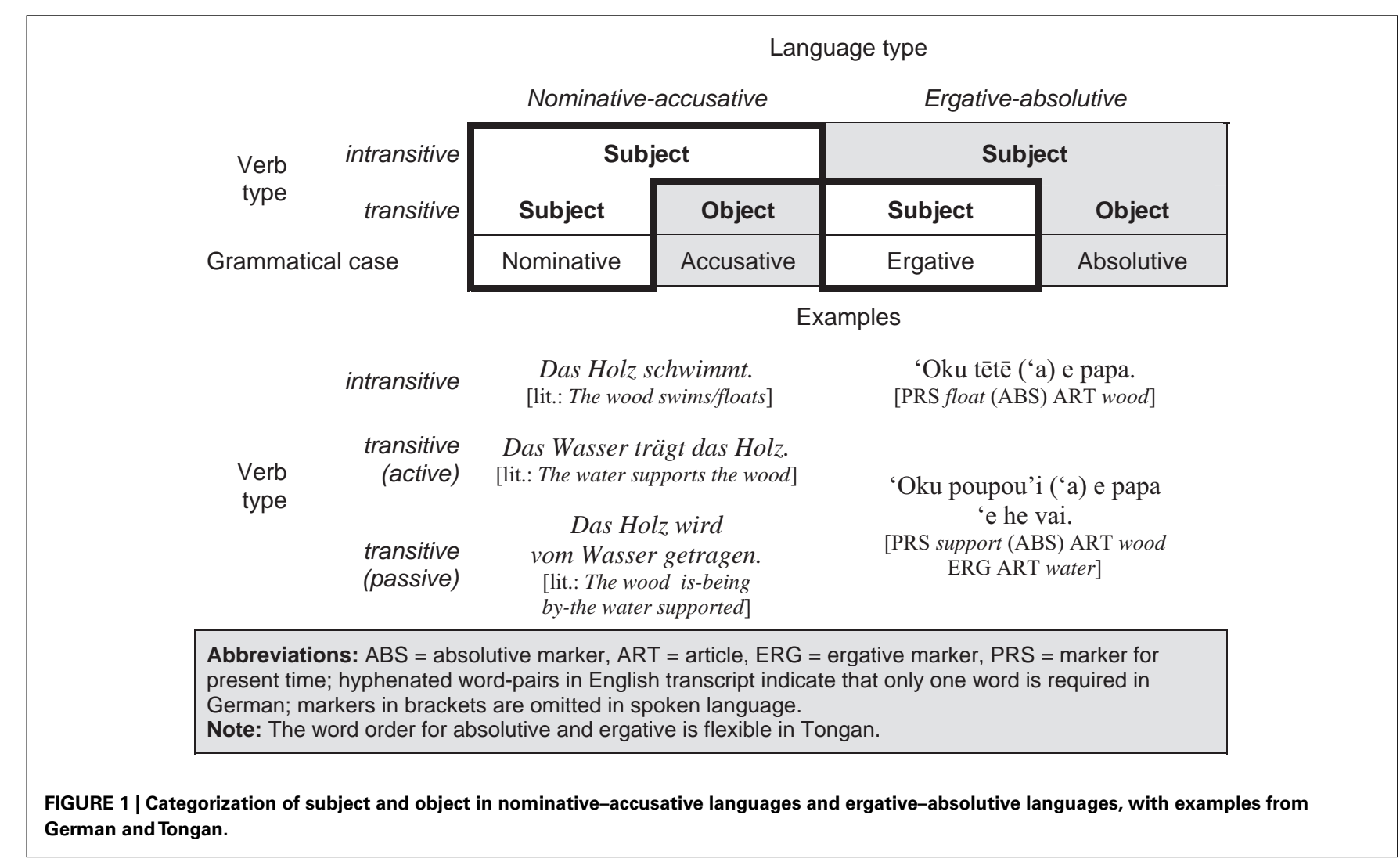


Beller, 2005; Beller and Bender, in preparation). If you receive the information that a piece of coal goes down in water whereas the piece of wood floats on water, you might consider the water as constant background and attribute the floating to the covarying entity wood (the figure) - although the floating of wood on water is completely independent of whether or not coal is floating. Changing the figure-ground mapping might thus be a powerful means for inducing a shift in causal role assignments.

Finally, the causal assignments might also depend on specific content concepts that people associate with the content of the task, giving rise to content effects (cf. Beller and Kuhnmünch, 2007; Beller et al., 2009b). As a priori assumptions are difficult to make with regard to which content elements induce which effect, we probed different kinds of elements: different physical relations (e.g., floating, rising vs. staying down, repelling vs. attracting) with different kinds of entities (e.g., freshwater floating on saltwater, wood floating on water, and cornflakes floating on milk). Therefore, this part of variation is rather explorative in nature. However, based on the cultural characteristics of Tonga as compared to Germany, two specific hypotheses may be ventured. Both are derived from the large degree of heteronomy and the pronounced emphasis on (social) support in Tonga. This awareness for the external causes of behavior in the social domain may be extended to other domains, thus nourishing a tendency to ascribe generally more responsibility to the carrier than to the floater in the floating setting (cf. Beller et al., 2009a,b). We therefore assumed that, first, causal assignments in Tonga may stick with the carrier even under altered conditions. To test this, we added a setting in which freshwater (which had been the carrier previously) now figured as the floater, being supported by saltwater. If, as we expected for our German participants, freshwater serves as the basic reference point, the causal assignment of our Tongan participants should switch from the carrier to the floater. If, however, the support aspect is more salient for them, causal assignment should still favor the carrier. And second, we assumed that asymmetric assignments among Tongans should be less pronounced for settings in which no single entity can be regarded as supporting the other. To test this, we added settings with even more symmetric relation, namely mutual attraction and dispersion.

\section{EXPERIMENT}

Nine physical settings were used in this study, six of which are concerned with buoyancy in different ways, two with dispersion, and one with gravitational attraction. Each setting mentions two entities that are equally involved for bringing about an effect. Thus, from a physical point of view, all settings are entirely symmetric, which is necessary in order to pin point asymmetric preferences in causal role assignments. In all tasks, people had to decide which of the two entities is responsible for bringing about the specific outcome.

\section{METHOD}

\section{Materials}

Nine physical settings were constructed according to the sentence frame that was used in the introductory floating task: "The fact that wood \{entity 1$\}$ floats on \{physical relation $\}$ water $\{$ entity 2$\}$ is basically due to ... the wood $\{$ entity 1$\} \ldots$ the water $\{$ entity
2\}." For each setting, different task versions were prepared by varying either agency, or the figure-ground mapping, or the content. An overview of the settings and of the experimental variations is given in Table 1 together with one example item from each setting.

Three settings are concerned with buoyancy in static floating events. In setting (A), we introduced a causative to shift agency from the floater ("freshwater floats on saltwater") to the carrier ("saltwater lets freshwater float"). In setting (B), we used a different verb to mark the carrier as agentive ("wood is supported by water") as compared to the floater ("wood floats on water"). This substitution of verbs coincided with a shift in voice (passive vs. active) in German and a shift from an ergative construction to a non-ergative construction in Tongan. In setting (C), we varied which entity is marked as figure: either the floater ("Cornflakes are placed in a bowl containing milk. The fact that ..."), or the carrier ("Milk is placed in a bowl containing cornflakes. The fact that ...") or none ("There are cornflakes and milk [milk and cornflakes] in a bowl. The fact that ...”).

Three settings are concerned with buoyancy in dynamic events. In setting (D), we probed the content of the entities by comparing concrete entities (" $\mathrm{CO}_{2}$ rises in water") with abstract entities ("a gas rises in a substance"). In setting (E), the same type of content manipulation was combined with a different verb: "staying down" in a concrete version (" $\mathrm{CO}_{2}$ stays down in air") and in an abstract version ("a gas stays down in a substance"). For setting (F), we reused the formulations of setting (E), both concrete and abstract, and added covariation information in order to mark as the figure of the scene either the gases (e.g., in the concrete version: "Helium rises in air. The fact that $\mathrm{CO}_{2}$ stays down in air ...") or the medium (e.g., " $\mathrm{CO}_{2}$ rises in water. The fact that $\mathrm{CO}_{2}$ stays down in air ...").

The last three settings are concerned with mutual attraction. Two dispersion events probed agency by varying the thematic role: In setting $(G)$, the verb "to mix" was used, focusing either on vinegar as agentive ("vinegar does not mix with oil"), or on oil ("oil does not mix with vinegar"), or on both in a linguistically symmetric way ("vinegar and oil [oil and vinegar] do not mix with each other"). In setting $(\mathrm{H})$, the verb "to repel" was used in the same way, focusing either on water as agentive ("water repels fat"), or on the fat ("fat repels water"), or symmetrically on both ("water and fat [fat and water] repel each other"). Finally, in setting (I) on gravitational attraction, the content of the entities was varied by comparing an abstract description with explicit size information ("a large and a small [a small and a large] celestial body attract each other") to concrete entities with implicit size information ("earth and moon [moon and earth] attract each other").

For each setting, participants were required to decide which of the two entities mentioned is responsible for bringing about the specific outcome. As the Tongan school system is based on a British curriculum, it can be assumed, that both Germans and Tongans have comparable formal knowledge of how the physical entities referred to in our settings relate to each other. Frequently, however, such formal knowledge only partly overwrites naïve, folk-physical convictions (e.g., McCloskey, 1983; Hubbard and Favretto, 2003). To outmaneuver possible effects of school education and tap into deeper, folk-theoretical convictions, participants were requested to decide between the two entities (in all settings 
Table 1 | The nine physical settings, the experimental variation under scrutiny, and one example item.

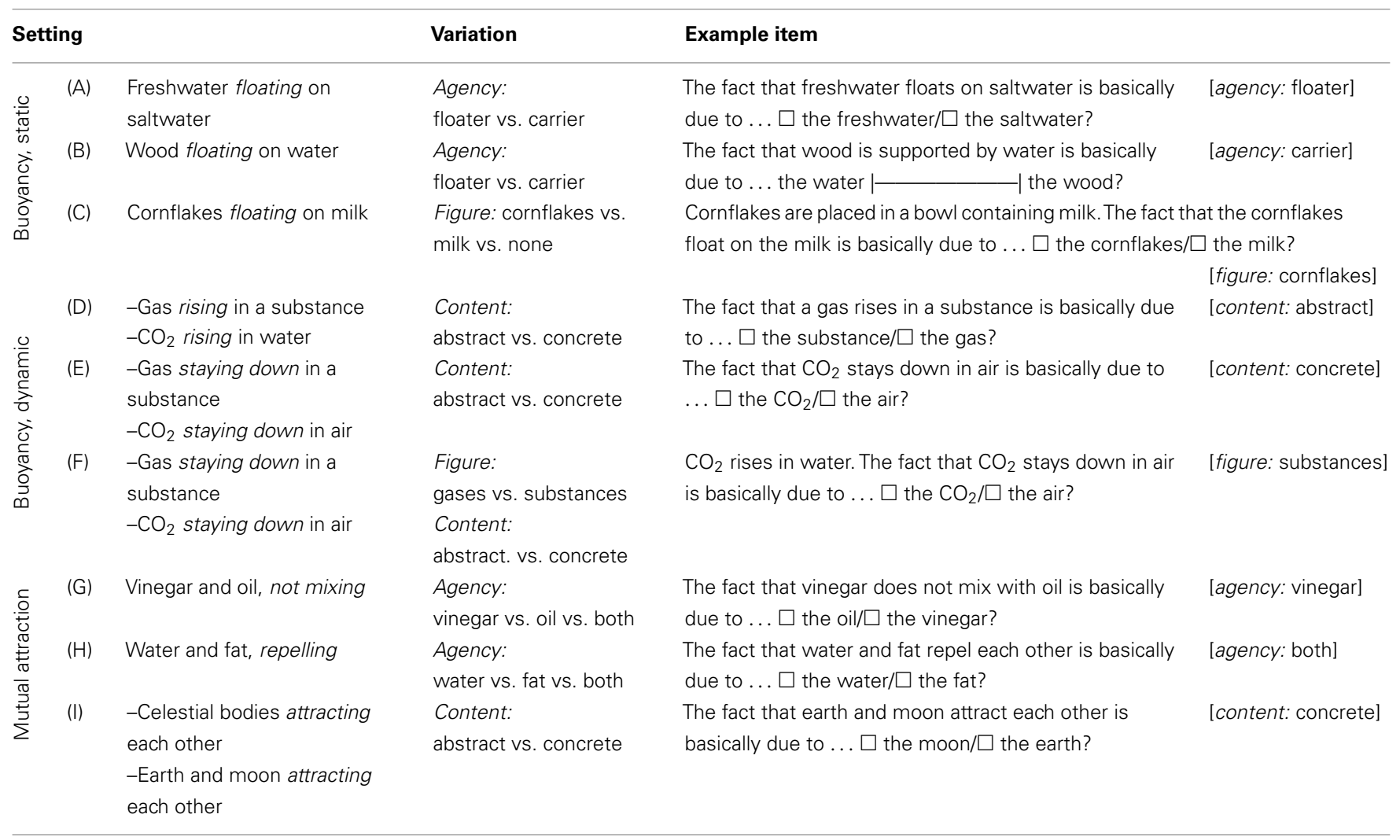

but setting B). If participants hold no such convictions (or if their convictions are compatible with the physical explanation), forcedchoices should trigger a random response pattern ceteris paribus, and thus result in a pattern that is balanced for both options. If one option is favored, an asymmetric pattern emerges, which may result from culture-specific concepts activated during processing, or from culture-specific patterns in the processing itself. To be able to assess whether the forced-choice format skews people's responses in an unintended way, setting (B) employed an analog rating scale (of $10 \mathrm{~cm}$ length) that allowed relative causal effectiveness to be allocated.

All materials were presented in the participants' native language (they had been translated by bilinguals and double checked in repeated re-translation sequences).

\section{Participants}

The German sample consisted of 93 students ( 36 male, 56 female) from the University of Freiburg with different fields of study (yet, excluding physics) and mean age 23.7 years $(S D=5.19)$. The Tongan sample consisted of 103 students (43 male, 56 female) from the Ha'apai High-School with mean age 16.9 years $(\mathrm{SD}=2.40)$.

Despite the difference in age, the participants are roughly comparable in terms of education level: The Tongan students attended a competitive high-school with Cambridge International Examinations Curriculum and were shortly before their final exams that qualify for university entry; most German students were shortly after these exams (please note that, until recently, German students were generally older than most others when taking these exams). These differences in age and education will be picked up in the discussion. Importantly, the Tongan students do not differ substantially from their Tongan age mates in terms of cultural immersion, at least not regarding social values, norms, and relationships (despite their access to advanced formal education).

\section{Design and procedure}

The tasks were administered in a mixed design with the nine settings presented within-subjects and the variations of each setting between-subjects. The order of the settings was randomized, with the following exceptions: Setting (B) was always presented last, because its analog scale offered more leeway for assigning causality. Setting (F) was never presented before (E), as (E) provided the baseline for the covariation conditions in (F). Also (A) and (C) were not presented in a row due to their related content, and the same applied for (D) and (E), and for $(\mathrm{G})$ and $(\mathrm{H})$. In the linguistically symmetric versions, the two entities were presented in balanced order, as were the two answer options in each task across the conditions. Participants were randomly assigned to the conditions.

\section{RESULTS}

All settings are symmetric from a physical perspective, and in some versions of settings $(G)$ to $(I)$ we even emphasized this by linguistically assigning agency to both entities. If not affected by assignment biases, people should solve all tasks in the same way, namely with a 50:50 distribution. Members of both cultures should solve all tasks in a similar way if they are not affected by culture-specific concepts 
or linguistic cues. The data of each setting were first checked for differences between cultures and for effects of the experimental variations (by means of log-linear analyses, if not indicated otherwise), and then analyzed for asymmetries in causal roles. An overview of the results is presented in Figure 2.

\section{Buoyancy, static events}

The three settings concerned with buoyancy in static events indicated cross-cultural similarities and differences, depending on the content of the setting, and figure-ground effects, but no effects of agency. Setting (A) with freshwater floating on saltwater revealed neither a difference between countries $\left(G^{2}=0.75 ; d f=1\right.$; $N=193 ; p=0.385)$, nor an effect of agency $\left(G^{2}=2.77 ; d f=1\right.$; $p=0.096)$, nor an interaction $\left(G^{2}=0.27 ; d f=1 ; p=0.605\right)$.
Aggregated across both agency versions, clear asymmetries were detected: The German participants preferred the carrier as causative for the floating $\left(73.9 \%\right.$ saltwater; $\chi^{2}=21.04 ; d f=1$; $N=92 ; p<0.001)$, and so did the Tongans $(79.2 \%$ saltwater; $\left.\chi^{2}=34.47 ; d f=1 ; N=101 ; p<0.001\right)$.

In setting (B) with wood floating on water, an analysis of variance revealed a clear difference between countries $[F(1,95)=20.40 ; p<0.001]$, but again no effect of agency $[F(1,95)=1.63 ; p=0.205]$ and no interaction $[F(1,95)=0.07$; $p=0.797]$. Again, causal asymmetries were detected, but this time they point in different directions: Aggregated across both agency versions, the German participants preferred the floater as causative $(69.6 \%$ wood; $t=5.96 ; d f=46 ; p<0.001)$, whereas the Tongan participants again preferred the carrier $(62.8 \%$ water;

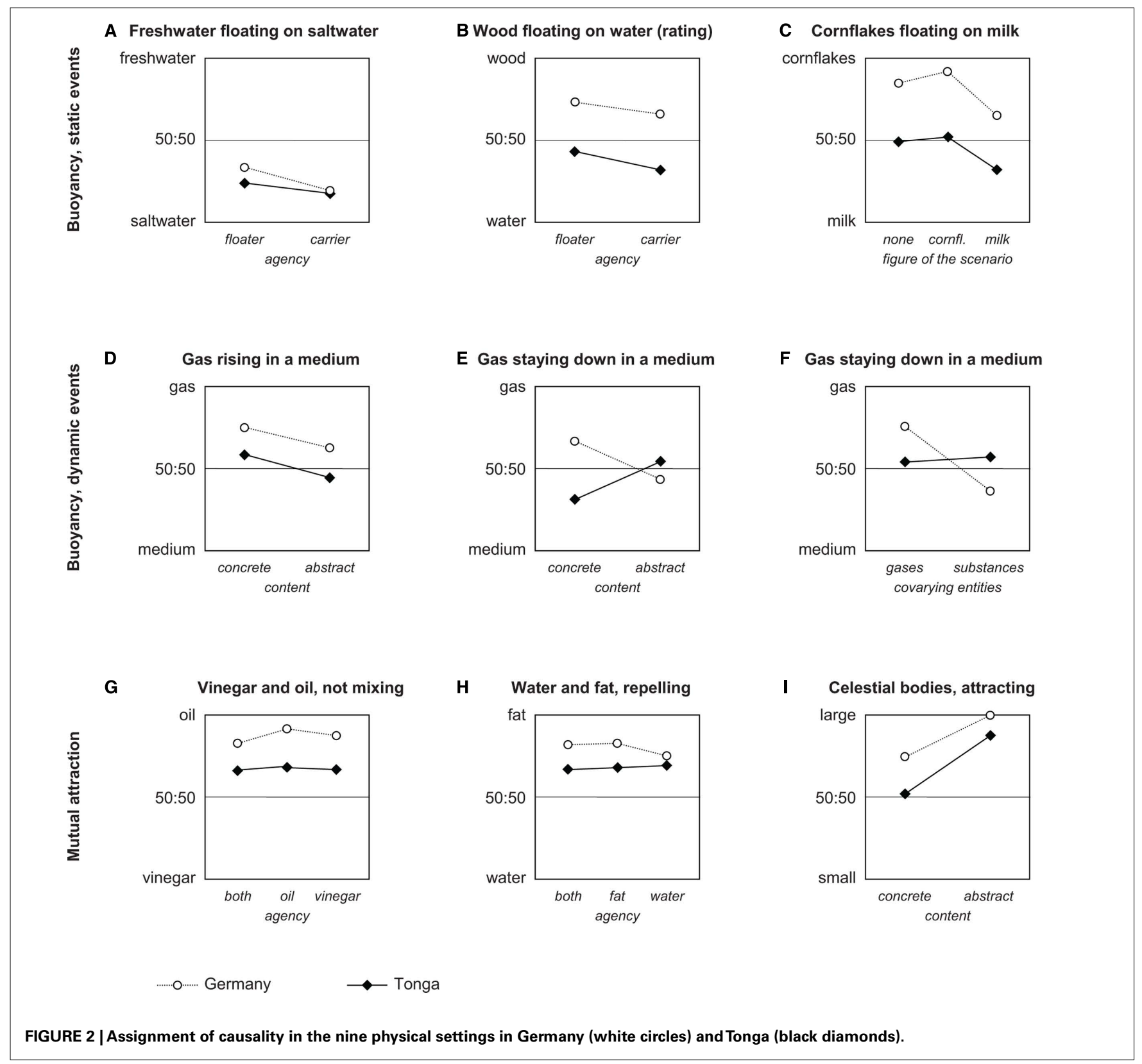


$t=-2.11 ; d f=51 ; p=0.040)$. The overall data pattern from this task replicates previous findings with this task despite two substantial changes of sampling and material (German university students and a rating format in this study vs. German high-school students and a forced-choice format in Beller et al., 2009b).

Finally, setting $(\mathrm{C})$ with cornflakes floating on milk revealed differences between countries $\left(G^{2}=28.23 ; d f=1 ; N=194\right.$; $p<0.001)$ and an effect of the figure-ground manipulation $\left(G^{2}=7.46 ; d f=2 ; p=0.024\right)$, but again no interaction $\left(G^{2}=0.85\right.$; $d f=2 ; p=0.653$ ). In the baseline condition (figure $=$ none), German participants preferred the floater as causative $(84.8 \%$ cornflakes; $\left.\chi^{2}=22.26 ; d f=1 ; N=46 ; p<0.001\right)$, whereas the Tongan participants revealed no clear preference $(49.0 \%$ cornflakes; $\left.\chi^{2}=0.02 ; d f=1 ; N=51 ; p=0.889\right)$. The preferences remained unchanged if the floater (cornflakes) was marked as the figure, but changed toward the carrier (milk) in both countries alike if the carrier was marked as the figure.

Comparing the three floating settings (A), (B), and (C), the data also indicate a clear content effect: In Germany, the preference shifts from the carrier as causative in setting (A) with freshwater floating on saltwater to the floater as causative in (B) and (C) with solid objects floating on a liquid; in Tonga, the supporting entities generally attracted more attention than in Germany.

\section{Buoyancy, dynamic events}

The three settings concerned with buoyancy in dynamic events indicated cross-cultural differences depending on the content and the figure of the setting in two of three cases. Setting (D) with gas rising in a substance indicated neither a difference between countries $\left(G^{2}=1.52 ; d f=1 ; N=97 ; p=0.218\right)$, nor an effect of content $\left(G^{2}=2.72 ; d f=1 ; p=0.099\right)$, nor an interaction $\left(G^{2}=0.002\right.$; $d f=1 ; p=0.964)$. Aggregated across both content versions, an asymmetry was detected in Germany $\left(68.8 \%\right.$ gas; $\chi^{2}=6.75 ; d f=1$; $N=48 ; p=0.009)$, but not in Tonga $\left(53.1 \%\right.$ gas; $\chi^{2}=0.184$; $d f=1 ; N=49 ; p=0.668)$.

Setting (E) with gas staying down in a substance did neither indicate a difference between countries $\left(G^{2}=1.64 ; d f=1 ; N=97\right.$; $p=0.200)$, nor an effect of content $\left(G^{2}=0.04 ; d f=1 ; p=0.840\right)$, but an interaction $\left(G^{2}=5.28 ; d f=1 ; p=0.022\right)$. Participants gave quite balanced answers in the abstract task $(43.5 \%$ gas in Germany and $54.2 \%$ gas in Tonga), but differed in the concrete task with a preference for the gas as causative in Germany $(66.7 \%$ gas; $\left.\chi^{2}=2.33 ; d f=1 ; N=21 ; p=0.127\right)$ and for the medium in Tonga (69.0\% medium; $\left.\chi^{2}=4.17 ; d f=1 ; N=29 ; p=0.041\right)$.

Setting (F) supplemented the contents from setting (E) with covariation information. As the content variation in $(\mathrm{F})$ did not make much of a difference, the data were pooled across concrete and abstract tasks. The analysis did not indicate differences between countries $\left(G^{2}=0.02 ; d f=1 ; N=194 ; p=0.887\right)$, but an effect of the figure-ground manipulation $\left(G^{2}=5.88 ; d f=1\right.$; $p=0.015)$, and an interaction $\left(G^{2}=9.07 ; d f=1 ; p=0.003\right)$. Tongan participants gave quite balanced answers irrespective of the covariation condition (54.0 vs. $56.9 \%$ gas; $\chi^{2}=0.08 ; d f=1$; $N=101 ; p=0.772)$. German participants preferred the gas as causative when the covariation pointed to the gases as figure, and the other substances (the medium) when the covariation pointed to the medium as figure (75.5 vs. $36.4 \%$ gas; $\chi^{2}=14.49$; $d f=1 ; N=93 ; p<0.001)$. As, from a physical point of view, the covariation information is irrelevant for the solution of this task, this latter finding is an instance of the distracting effect of covariation information that we found in Germany with other physical settings as well (Beller and Bender, in preparation).

\section{Mutual attraction}

The three settings concerned with mutual attraction indicated cross-cultural similarities in the assignment of causality and content effects, but again no effects of agency. Setting $(G)$ with vinegar and oil not mixing with each other revealed differences between countries $\left(G^{2}=10.28 ; d f=1 ; N=195 ; p=0.001\right)$, but no effect of agency $\left(G^{2}=0.53 ; d f=2 ; p=0.769\right)$ and no interaction $\left(G^{2}=0.57 ; d f=2 ; p=0.751\right)$. Aggregated across all three agency versions, clear asymmetries were detected: The German participants preferred oil as causative $\left(86.0 \%\right.$ oil; $\chi^{2}=48.27$; $d f=1 ; N=93 ; p<0.001)$, and so did the Tongan participants, albeit to a lesser degree $\left(66.7 \%\right.$ oil; $\chi^{2}=11.33 ; d f=1 ; N=102$; $p=0.001$ ).

Nearly the same result was found in the second dispersion setting $(\mathrm{H})$ with water and fat repelling each other: The analysis indicated differences between countries $\left(G^{2}=4.14 ; d f=1 ; N=194\right.$; $p=0.042)$, but again no effect of agency $\left(G^{2}=0.13 ; d f=2\right.$; $p=0.939)$, and no interaction $\left(G^{2}=0.52 ; d f=2 ; p=0.773\right)$. Aggregated across the three agency versions, similar asymmetries as in setting $(\mathrm{G})$ were detected: The German participants preferred the fat as causative $\left(80.4 \%\right.$ fat; $\chi^{2}=34.09 ; d f=1 ; N=92$; $p<0.001)$, and so did the Tongan participants, albeit to a lesser degree $\left(67.6 \%\right.$ fat $\left.; \chi^{2}=12.71 ; d f=1 ; N=102 ; p<0.001\right)$.

Finally, setting (I) concerning gravitational attraction between celestial bodies revealed differences between countries $\left(G^{2}=6.35\right.$; $d f=1 ; N=117 ; p=0.012)$ and an effect of content $\left(G^{2}=18.86\right.$; $d f=1 ; p<0.001)$, but no interaction $\left(G^{2}=2.28 ; \quad d f=1\right.$; $p=0.131)$. In the abstract task, the larger celestial body was preferred as causative in Germany (100.0\% large; $p<0.001$ according to the binomial distribution with $r=1 / 2$ and $N=31)$ as well as in Tonga ( 87.5 large; $\left.\chi^{2}=13.50 ; d f=1 ; N=24 ; p<0.001\right)$. In the concrete task, the larger body (the earth as compared to the moon) was still preferred in Germany $\left(74.2 \%\right.$ large; $\chi^{2}=7.26 ; d f=1$; $N=31 ; p=0.007)$, but not in Tonga $\left(51.6 \%\right.$ large; $\chi^{2}=0.03$; $d f=1 ; N=31 ; p=0.857)$.

\section{General patterns}

Summarizing the data, four general findings emerge: First, causal asymmetries were found in both cultures (in eight of the nine settings in Germany and in six settings in Tonga), but they varied across tasks and cultures. In four settings (A, G, H, and I), the biases were alike, in one setting (B) they diverged, and in four settings ( $C, D, E$, and $F$ ), a bias was detected in one culture only. With two exceptions (A and $\mathrm{E}$ ), the asymmetry was generally less pronounced in Tonga, as was expected due to the general cultural orientation, which is more collectivistic in Tonga than in Germany. Second, the linguistic variations of agency (employed in settings $\mathrm{A}, \mathrm{B}, \mathrm{G}$, and $\mathrm{H}$ ) had no effect on participants' causal assignments - contrary to what we expected. Third, whichever object was focused on as the figure of the setting was given the stronger weight, as predicted ( $\mathrm{C}$ and $\mathrm{F}$ in Germany, and $\mathrm{C}$ in Tonga). Finally, 
the asymmetries were modulated by the task content: Its strength and direction varied across physical relations (cf. rising in $\mathrm{D}$ and staying down in $\mathrm{E}$ ) and also across the entities involved (e.g., in the three floating settings $\mathrm{A}, \mathrm{B}$, and $\mathrm{C}$ ). Abstracting from concrete entities had diverging effects: The abstract versions were less prone to asymmetric assignments in the buoyancy tasks (D and E), where abstraction highlights the (symmetric) physical relation, but more so in the attraction task (I), where the abstraction co-occurs with an explication of the size of the entities.

Compared to the German participants, our Tongan participants seem to be generally less susceptible to assignment biases, at least on the group level. But does this also hold on the individual level? To answer this question, we analyzed the distributions of the rating data pooled for the two agency conditions (see Figure 3). While in Germany, the mean asymmetry toward the floater is reflected in the distribution of the individual ratings, which is skewed to the right (skewness $=-0.817 ; \mathrm{SE}=0.347$ ), the more balanced mean rating in Tonga is not at all reflected in the distribution (skewness $=0.576$; $\mathrm{SE}=0.330$ ). Rather, the Tongan pattern is marked by two clear peaks - a stronger one on the carrier and a weaker one on the floater - while barely anybody gave a balanced rating. A symmetric pattern on the group level in the forced-choice format does therefore not speak against an assignment bias among individuals. This also implies that this format provides a rather conservative test for asymmetries, which are only attested if people consistently exhibit the same preference. Importantly, this data pattern also speaks against a simple explanation in terms of an individualistic vs. collectivistic orientation: The individualism/collectivism paradigm predicts that members of a culture with a strong collectivistic orientation should consider circumstances more strongly and thus assign causality in a more balanced manner than members of an individualistic culture. As seen above, on the group level this was indeed observed for Tonga and Germany. On the individual level, however, Tongans tended to assign causality in a less balanced manner than Germans.

\section{DISCUSSION}

Our findings provide cross-cultural evidence for asymmetric causal role assignments in physically symmetric settings and thereby go beyond previous research in three respects: First, while concurring with White's findings of a pervasive causal asymmetry

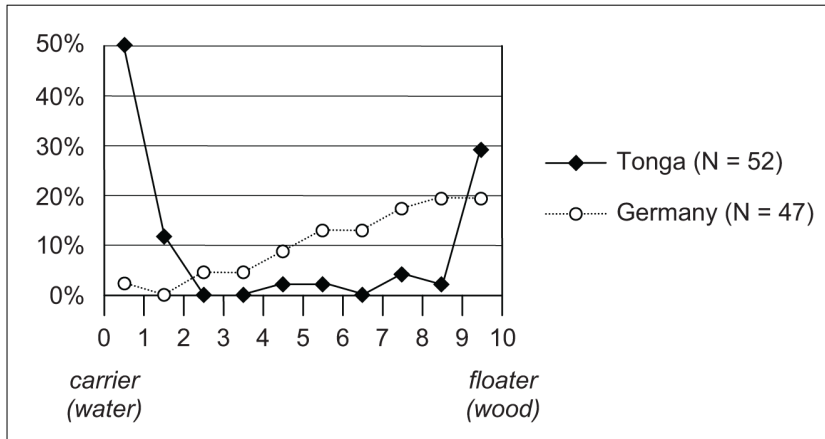

FIGURE 3 | Distribution of the causal assignments in setting B with the analog rating format (in decentiles), pooled across the two agency conditions. bias in physical settings, they demonstrate assignment preferences even for static settings that are discernibly more symmetric than the original launching setting used by White (2007). However, we hesitate to interpret our findings as evidence for the causal asymmetry bias in the narrower sense, as we tested only one of the two stages White (2006) considers prerequisite: We did identify which factor people perceive as causative, but we did not separately assess its assumed importance. However, at least on the aggregated level, assigning causal roles results in asymmetric patterns (even for unquestionably symmetric relations) that betray an underlying bias. Second, our findings document such assignment preferences in two very different cultures. This does not prove, in a strict sense, that this phenomenon is universal, but it is a first piece of evidence in this direction (Norenzayan and Heine, 2005). And third, they show that the strength and occasionally even the direction of the asymmetry can be modulated in various ways, thus resulting in culture-specific and task-specific preferences.

While these findings concur, in general, with Peng and Knowles' (2003) conclusion that culture affects causal cognition, it goes beyond the classic focus on "East-West" differences in general attribution tendencies in two ways. First, they reveal that compatibility of causal assignments with general attribution tendencies on the group level need not be reflected on the individual level. And second, our study identified two additional factors for people's assignments: figure-ground mapping and task content. While figure-ground mapping should (and did) have similar effects in both cultures, a priori assumptions with regard to how culturespecific concepts will affect causal assignments are not easily put forward. However, the findings of the static floating settings (A, $\mathrm{B}$, and $\mathrm{C}$ ) are in line with our (preliminary) hypothesis that the strong Tongan focus on cooperation and support might extend to the physical domain: In Tonga, supporting entities did attract more attention - saltwater in (A), water in (B), and milk in (C). In Germany, on the other hand (fresh-)water and other drinkable liquids seem to provide the background against which other substances - saltwater in setting (A), wood in (B), and cornflakes in (C) - are then considered specific and hence causative. Far from confirming the previously widespread assumption that appraising physical causality should be objective and largely independent of culture, our data thus attest to a profound susceptibility of causal cognition to a range of factors, even in simple physical settings.

Three reservations have to be made, though, with regard to the generality of our results. First, the cultural differences we found crucially depend on how comparable our samples were. As stated in the Section "Participants," we believe their educational background to be not too different, while mean age clearly is. One of the settings allows us to assess, to some extent, whether this difference may have had substantial effects: Setting (B) "wood floats on water" was also used in a related study (Beller et al., 2009b) with German and Tongan high-school students not only of the same educational background but also of the same age, and this study obtained nearly identical results, namely a preference for the carrier in Tonga and for the floater in Germany. This replication with different samples encourages us to interpret the differences obtained in the current study as indeed arising from culture-specific factors. Additional support for this interpretation 
stems from the fact that differences between Tongan and German participants were not constant across tasks. Response patterns differed in some settings only, while in others they resembled each other (e.g., in settings A, G, and H), thus suggesting contentspecific interpretations of each task. Moreover, the forced-choice options are not compatible with whatever our participants may have learned during formal education, neither in Germany nor in Tonga. This was explicitly intended to tap deeper folk-physical intuitions (which can be assumed to be only partly overwritten by school knowledge). And indeed, even where it was possible to indicate the physically correct solution on the analog rating scale, only a few participants chose to do so. We hold, therefore, that the observed occurrence of a strong assignment bias in this regard may be interpreted as reflecting underlying folk-physical convictions. Nevertheless - and beyond the question of sample comparability - it would be highly desirable to replicate our screening study with more diverse samples in both cultures.

The second reservation concerns our usage of the forced-choice format. This was considered legitimate for the purpose of this study as it prevented participants from simply activating the formal knowledge acquired in school. As we controlled for all factors that might shift participants' attention (such as the order in which the response options occurred or the order in which entities in symmetric settings were mentioned), we can exclude that the accumulation of answers was an artifact. Rather, it can be interpreted as reflecting some prevailing tendency. In support of this argument, a comparison of two identical tasks on the same content - one with forced-choice (Beller et al., 2009b) and one with rating scale (this study, setting B) - yielded no significant differences in response patterns of German or Tongan participants. However, given that task content partly modulated people's responses, it is difficult to generalize from just one task to the whole set. Furthermore, being able to assess more faithfully the actual convictions and conceptual models of participants would be highly desirable for future studies. One option to achieve this could be to ask participants for explanations. This would also help to tackle the important question of which of the two potentially competing concepts (i.e., the scientific ones learned in formal education and the folk-theoretical ones) is actually more relevant for how people understand the world and respond to it (cf. Legare and Gelman, 2008; Legare et al., in press). So far, we could show that our participants do access folk-theoretical concepts under certain circumstances, but we cannot infer in which real-world contexts they indeed prefer those concepts.

And finally, other than in the previous study (Beller et al., 2009b), the different versions of cueing agency adopted in this survey yielded no significant effect. In particular, the intended shift in focus enclosed in the variation of "A floats on B" vs. "B lets A float" did not affect the response pattern in either language. The

\section{REFERENCES}

Arnett, J. J. (2008). The neglected 95\%: why American psychology needs to become less American. Am. Psychol. 63, 602-614.

Atran, S., and Medin, D. L. (2008). The Native Mind and the Cultural
Construction of Nature. Boston: MIT Press.

Beller, S., and Bender, A. (2008). The limits of counting: numerical cognition between evolution and culture. Science 319, 213-215.

same holds for the variation of "is floating" vs. "is supported by." However, as these two sets of formulations do not cover the whole range of possible formulations, this does not preclude that linguistic cueing may have effects more generally. The (linguistically induced) figure/ground reversal provides a case in point, where such manipulations were indeed effective. Clearly, more in-depth analyses and a broader range of linguistic variants are required here to settle this question.

Our findings have important implications. Asymmetric assignment of causal roles leads people to perceive, understand, and describe interactions not as symmetric relations, but as "relations between doer and done-to" (White, 2006). Once people have decided which entity they regard as causative, they tend to overestimate its importance. Yet, focusing on single factors as critical may impair problem solving, for instance with regard to technical malfunctioning, ecosystem management, or social conflicts; and even in simple physical settings, it will at least impede learning and understanding. Analyzing the consequences of such assignment biases for the causal reasoning processes of both laypersons and scientists thus remains a crucial task for the future.

In doing so it also needs to be kept in mind that "culture" is not simply a homogenous entity that may lead in just one direction. Instead, people's cultural background is composed of innumerous different components. Owing to this multifaceted character, its impact on causal cognition may give rise to heterogeneous patterns. As we have seen in this study, asymmetric assignments are triggered not by one single rationale, but by a host of different aspects (e.g., the physical relation under scrutiny, the concrete entities involved, the figure-ground distinction, etc.). The general mechanism involves processes of activating, selecting, and weighing up relevant information - but we have only just begun to explore this conglomeration. More research is required to identify the specific concepts upon which people base their assessments and to explore the ways in which they acquire them. Finding cultural differences therefore cannot be the end of the endeavor, but need to be understood as starting point (Bender et al., 2010b; Bender and Beller, 2011b). If we are striving to achieve a comprehensive picture of human cognition, it is indispensable to take its cultural constitution into account.

\section{ACKNOWLEDGMENTS}

We thank the government of Tonga for granting us research permission, and we are grateful to Christoph Klauer, Toka Puleiku, and Moana Faka'osi for support with data collection, and to Susanne Bubser, Sione Faka'osi, Sarah Mannion de Hernandez, and the two reviewers for valuable comments on earlier versions of this paper. The article processing charges are covered by the Publication Fund of the Library of Freiburg University, supported by a grant of the Deutsche Forschungsgemeinschaft DFG.

Beller, S., Bender, A., and Song, J. (2009a). Conditional promises and threats in Germany, China, and Tonga: cognition and emotion. J. Cogn. Cult. 9, 115-139.

Beller, S., Bender, A., and Song, J. (2009b). Weighing up physical causes: effects of culture, linguistic cues, and content. J. Cogn. Cult. 9, 347-365.

Beller, S., and Kuhnmünch, G. (2007). What causal conditional reasoning tells us about people's understanding 
of causality. Think. Reas. 13, 426-460.

Beller, S., and Spada, H. (2003). The logic of content effects in propositional reasoning: the case of "conditional reasoning with a point of view." Think. Reas. 9, 335-378.

Bender, A. (2001). "God will send us the fish": perception and evaluation of an environmental risk in Ha'apai, Tonga. Res. Soc. Probl. Public Policy 9, 165-190.

Bender, A., and Beller, S. (2003). "Polynesian tapu in the 'deontic square': a cognitive concept, its linguistic expression and cultural context," in Proceedings of the 25th Annual Conference of the Cognitive Science Society, eds R. Alterman and D. Kirsh (Mahwah, NJ: Lawrence Erlbaum Associates), 133-138.

Bender, A., and Beller, S. (2011a). Cultural variation in numeration systems and their mapping onto the mental number line. J. Cross Cult. Psychol. 42, 579-597.

Bender, A., and Beller, S. (2011b). The cultural constitution of cognition: taking the anthropological perspective. Front. Psychol. 2:67. doi: 10.3389/fpsyg.2011.00067

Bender, A., Beller, S., and Bennardo, G. (2010a). Temporal frames of reference: conceptual analysis and empirical evidence from German, English, Mandarin Chinese, and Tongan. J. Cogn. Cult. 10, 283-307.

Bender, A., Hutchins, E., and Medin, D. L. (2010b). Anthropology in cognitive science. Top. Cogn. Sci. 2, 374-385.

Bender, A., Spada, H., Seitz, S., Swoboda, H., and Traber, S. (2007). Anger and rank in Tonga and Germany: cognition, emotion, and context. Ethos 35, 196-234.

Boroditsky, L., and Gaby, A. (2010). Absolute spatial representations of time in an Aboriginal Australian community. Psychol. Sci. 21, 1635-1639.

Cheng, P. W. (1997). From covariation to causation: a causal power theory. Psychol. Rev. 104, 367-405.

Chung, S. (1978). Case Marking and Grammatical Relations in Polynesian. Austin, TX: University of Texas Press.

Cummins, D. D. (1995). Naive theories and causal deduction. Mem. Cognit. 23, 646-658.

Duranti, A. (1994). From Grammar to Politics: Linguistic Anthropology in a Western Samoan Village. Berkeley, CA: University of California Press.

Evans, M. (2001). Persistence of the Gift. Waterloo: Wilfrid Laurier University Press.

Fausey, C., and Boroditsky, L. (2011). Who dunnit? Cross-linguistic differences in eye-witness memory. Psychon. Bull. Rev. 18, 150-157.

Gopnik, A., and Schulz, L. (eds). (2007) Causal Learning. Oxford: Oxford University Press.

Henrich, J., Heine, S. J., and Norenzayan, A. (2010). The weirdest people in the world? Behav. Brain Sci. 33, 1-75.

Hubbard, T. L., and Favretto, A. (2003). Naïve impetus and Michotte's "tool effect": evidence from representational momentum. Psychol. Res. 67, 134-152.

Kempton, W. M. (1986). Two theories of home heat control. Cogn. Sci. 10, 75-90.

Klauer, K. C., Beller, S., and Hütter, M. (2010). Conditional reasoning in context: a dual-source model of probabilistic inference. J. Exp. Psychol. Learn. Mem. Cogn. 36, 298-323.

Kuhnmünch, G., and Beller, S. (2005). Distinguishing between causes and enabling conditions - through mental models or linguistic cues? Cogn. Sci. 29, 1077-1090.

Legare, C. H., Evans, E. M., Rosengren, K., and Harris, P. (in press). The coexistence of natural and supernatural explanations across cultures and development. Child Dev.

Legare, C. H., and Gelman, S. A. (2008). Bewitchment, biology, or both: the co-existence of natural and supernatural explanatory frameworks across development. Cogn. Sci. 32, 607-642.

Majid, A., Bowerman, M., Kita, S., Haun, D. B. M., and Levinson, S. C. (2004).
Can language restructure cognition? The case for space. Trends Cogn. Sci. (Regul. Ed.) 8, 108-114.

Majid, A., Sanford, A. J., and Pickering, M. J. (2007). The linguistic description of minimal social scenarios affects the extent of causal inference making. J. Exp. Soc. Psychol. 43 , 918-932.

Malle, B. (1999). How people explain behavior: a new theoretical framework. Pers. Soc. Psychol. Rev. 3, 23-48.

Marcus, G. E. (1978). Status rivalry in a Polynesian steady-state society. Ethos 6, 242-269.

Markus, H. R., and Kitayama, S. (1991). Culture and the self: implications for cognition, emotion, and motivation. Psychol. Rev. 98, 224-253.

McCloskey, M. (1983). Intuitive physics. Sci. Am. 248, 122-130.

Michotte, A. (1963). The Perception of Causality. New York: Basic Books.

Morris, M. W., and Peng, K. (1994). Culture and cause: American and Chinese attributions for social and physical events. J. Pers. Soc. Psychol. 67, 949-971.

Morton, H. (1996). Becoming Tongan: An Ethnography of Childhood. Honolulu, HI: University of Hawai'i Press.

Norenzayan, A., and Heine, S. J. (2005). Psychological universals: what are they and how can we know? Psychol. Bull. 131, 763-784.

Norenzayan, A., and Nisbett, R. E. (2000). Culture and causal cognition. Curr. Dir. Psychol. Res. 9, 132-135.

Peng, K., Ames, D. R., and Knowles, E. D. (2001). "Culture and human inference: perspectives from three traditions," in Handbook of Culture and Psychology, ed. D. R. Matsumoto (New York: Oxford University Press), 245-264.

Peng, K., and Knowles, E. D. (2003). Culture, education, and the attribution of physical causality. Pers. Soc. Psychol. Bull. 29, 1272-1284.

Spelke, E. S., and Kinzler, K. D. (2007). Core knowledge. Dev. Sci. 10, 89-96.
Sperber, D., Premack, D., and Premack, A. J. (eds). (1995). Causal Cognition. Oxford: Clarendon Press.

Talmy, L. (1988). Force dynamics in language and cognition. Cogn. Sci. 12, 49-100.

Waldmann, M. R., Hagmayer, Y., and Blaisdell, A. P. (2006). Beyond the information given: causal models in learning and reasoning. Curr. Dir. Psychol. Res. 15, 307-311.

White, P. A. (2006). The causal asymmetry. Psychol. Rev. 113, 132-147.

White, P. A. (2007). Impressions of force in visual perception of collision events: a test of the causal asymmetry hypothesis. Psychon. Bull. Rev. 14, 647-652.

Wolff, P. (2007). Representing causation. J. Exp. Psychol. Gen. 136, 82-111.

Wolff, P., Klettke, B., Ventura, T., and Song, G. (2005). "Expressing causation in English and other languages," in Categorization Inside and Outside of the Laboratory, eds W. Ahn, R. L. Goldstone, B. C. Love, A. B. Markman, and P. Wolff (Washington, DC: APA), 29-48.

Conflict of Interest Statement: The authors declare that the research was conducted in the absence of any commercial or financial relationships that could be construed as a potential conflict of interest.

Received: 22 July 2011; accepted: 29 August 2011; published online: 22 September 2011.

Citation: Bender $A$ and Beller $S$ (2011) Causal asymmetry across cultures: assigning causal roles in symmetric physical settings. Front. Psychology 2:231. doi: 10.3389/fpsyg.2011.00231

This article was submitted to Frontiers in Cultural Psychology, a specialty of Frontiers in Psychology.

Copyright (c) 2011 Bender and Beller.

This is an open-access article subject to a non-exclusive license between the authors and Frontiers Media SA, which permits use, distribution and reproduction in other forums, provided the original authors and source are credited and other Frontiers conditions are complied with. 